\title{
Research on Polyethylene Glycol, Crosslinked Polyethylene Glycol \& Polyethylene Glycol Chitosan Conjugate Coating for Biomedical Application
}

\begin{abstract}
Polyethylene glycol used as coating materials because it resist to protein adsorption and bacterial adhesion. Polyethylene glycol and crosslinked polyethylene glycol \& polyethylene glycol chitosan conjugate used for coating glass implants. Fourier transform infrared spectroscopy (FTIR) analysis showed proper crosslinking in crosslinked polyethylene glycol but in polyethylene glycol \& chitosan conjugate no reaction takes place. Scanned Electron Microscope (SEM) used for study of surface morphology. Polyethylene glycol \& chitosan conjugate have smoother surface than pure polyethylene glycol, polyethylene glycol chitosan conjugate. Differential scanning calorimetry (DSC) analysis used for glass transition temperature study. Crosslinked polyethylene glycol has good mechanical properties. Antimicrobial properties of polyethylene glycol chitosan conjugate are good in comparison to pure polyethylene glycol, crosslinked polyethylene glycol.
\end{abstract}

Sarita

Keywords: Chitosan, Polyethylene glycol (PEG), Antimicrobial properties, Mechanical testing, Thermal properties.

\section{INTRODUCTION}

Biomaterials are widely used in many implants and medical associated devices; to be implanted inside human body it should have excellent bulk as well as surface properties. The use of these medical devices has lead to new field of revolution in field of medicine. Increased use of these devices has not only increased the life expectancy of patient but also resulted in better quality of life. But one of the major barriers in long term usage of these devices has micro infection. The only solution to this problem is removal of implants which increase patient mortality and compliance. It is in this regard that surface properties of biomaterial should be modified so as to decrease adhesion of microbes, with increase antimicrobial properties and other beneficial properties. Surface properties which affects the function of biomaterials are mainly surface topology, surface charge etc [1]. Surface can be modified by coating, oxidation by low temperature plasma, surfactant addition for anti- static. Blending also uses for improving tensile properties and to provide a stronger structural component for separation media that supports the active polymer. Commonly used surface modification techniques include coating, oxidation by low-temperature plasma for better printing and adhesion and surfactant addition for anti-static.

Polyethylene glycol (PEG) is a water soluble polymer with

Revised Version Manuscript Received on 10, September 2019.

Sarita, Deen Bandhu Chottu Ram Uni. Of Sci. and Technology Murthal Sonipat, Haryana, India 131039 many molecular weights that exhibits useful properties such as protein resistance, low toxicity and immunogenicity. PEG shows good solubility in water, biocompatibility, and drug carrier. PEG can be copolymerised with any other polymer for improving its properties.

Chitosan is the $\mathrm{N}$-deacetylated derivative of chitin, a cationic polysaccharide composed of glucosamine and $\mathrm{N}$-acetylglucosamine residues with 1, 4-b-linkage [5]. Chitin polysaccharihide is one of the most abundant found in nature. PEG and chitosan both have excellent properties so they used

in combination for increase properties like mechanical and biocompatibility. Antimicrobial properties as well as thermal and mechanical properties examined for performance testing.

\section{MATERIALS AND METHOD}

Chitosan purchased from sigma, polyethylene glycol from hi media, acetic acid, gluteraldehyde, nutrient agar.

\subsection{Sample preparation}

Pure polyethylene glycol:

Pure polyethylene glycol prepared for coating by dissolving $400 \mathrm{mg}$ polyethylene glycol in to $5 \mathrm{ml}$ distilled water. Then stir the solution over night for proper mixing. Coat this sample on a glass slab

\section{Crosslinked polyethylene glycol:}

$5 \mathrm{ml}$ polyethylene glycol was dissolved in $5 \mathrm{ml}$ distilled water. After proper mixing add $s \mu l$ gluteraldehyde for crosslinking. Stir the solution over night after that coat it on glass.

\section{Polyethylene glycol and chitosan conjugate:}

$1 \mathrm{~g}$ of chitosan was dissolved in $100 \mathrm{ml}, 2 \%$ acetic acid solution after that add $8 \mathrm{ml}$ distilled water. Stir it till clear solution obtained. Then add $2 \mathrm{ml}$ polyethylene glycol and stir for overnight. 
Research on Polyethylene Glycol, Crosslinked Polyethylene Glycol \& Polyethylene Glycol Chitosan Conjugate Coating for Biomedical Application

\section{RESULT AND DISCUSSION}

\subsection{Antimicrobial testing:}

Antimicrobial testing has been done by agar diffusion method. Sample prepared for coating showed antimicrobial properties against E.coli. A clear zone of inhibition showed antimicrobial properties.

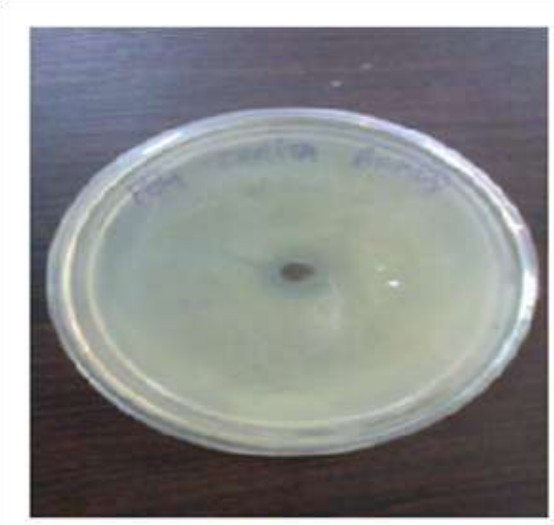

(a)

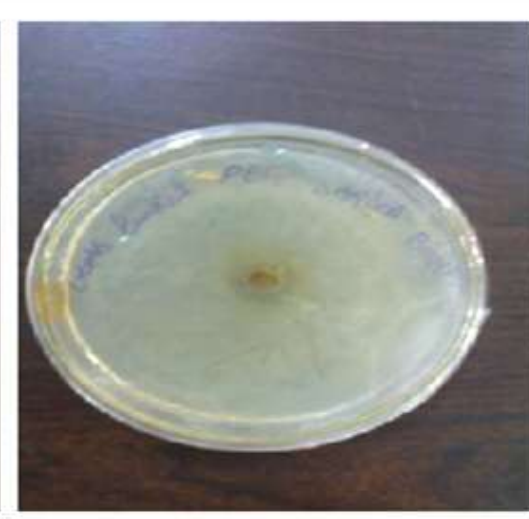

(b)

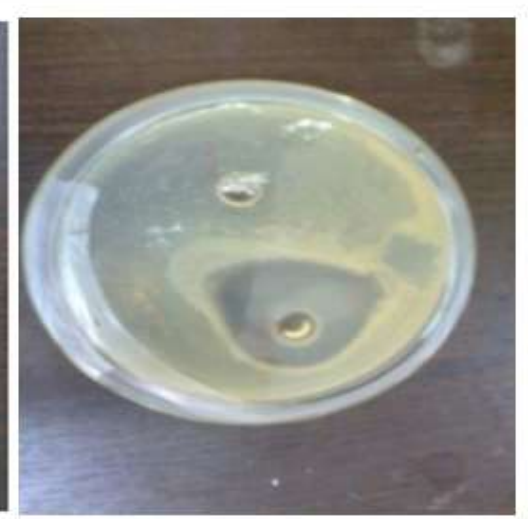

(c)

Fig. 1. (a) Antimicrobial properties of pure polyethylene glycol, (b) crosslinked polyethylene glycol, (c) Polyethylene glycol \& chitosan conjugate against $E$.coli

Zone of inhibition $(\mathrm{cm})$ of polyethylene glycol \& chitosan conjugate has large diameter than two other samples, which showed strong antimicrobial properties.

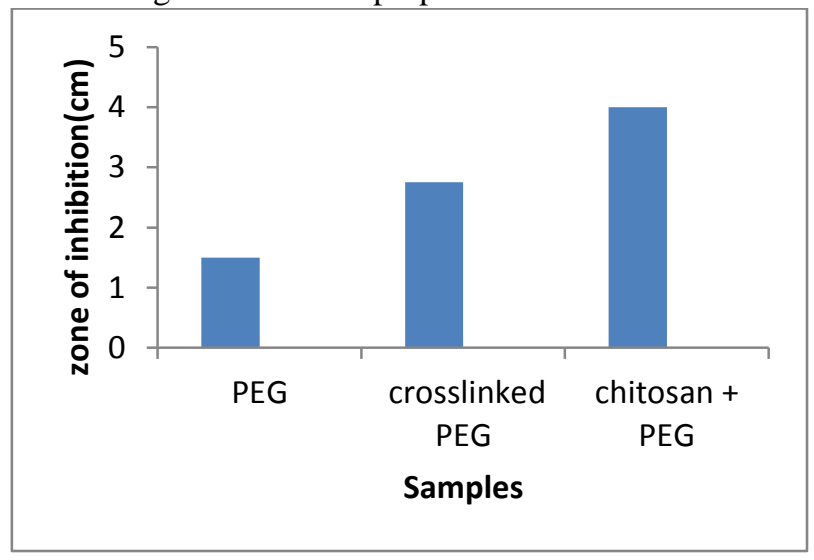

Fig.2. Comparison of zone of inhibition of coating samples

\subsection{Thermal analysis:}

DSC curve showed glass transition temperature of pure polyethylene glycol which is $65^{\circ} \mathrm{c}$. Crosslinked polyethylene glycol has not any sharp peak due to moisture content, crosslinker [5]. Polyethylene glycol \& chitosan have melting point $65^{\circ} \mathrm{C}$.

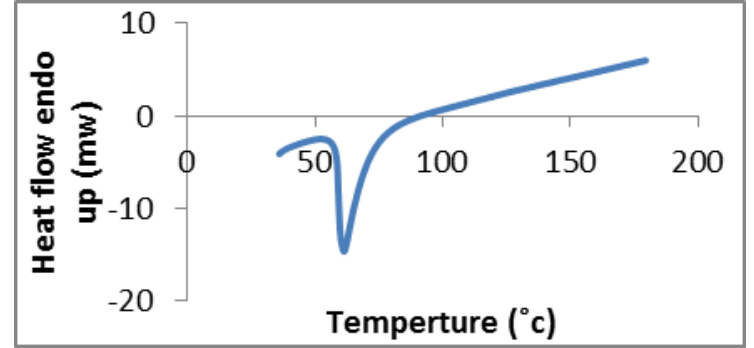

(a)

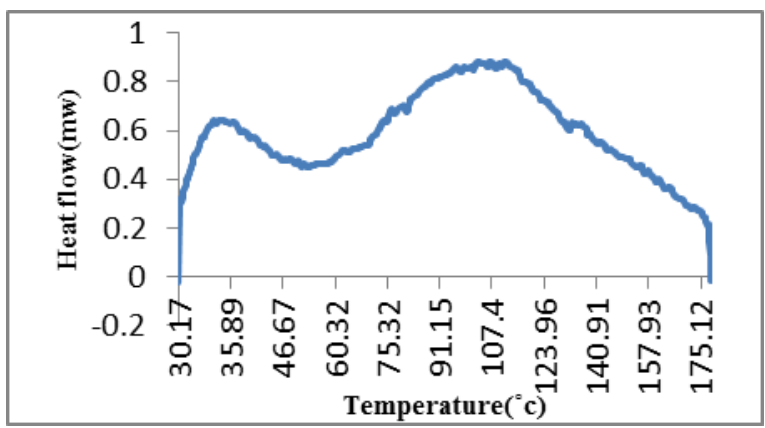

(b)

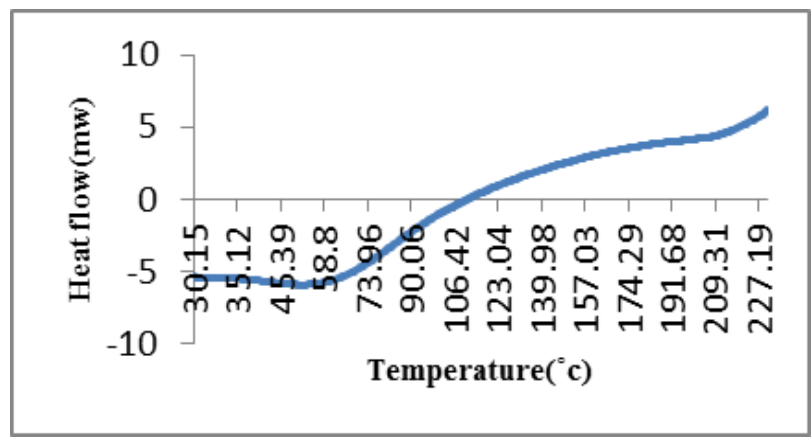

(c)

Fig.3. (a) DSC curve of pure polyethylene glycol, (b) Crosslinked polyethylene glycol, (c) polyethylene glycol \& chitosan conjugate 


\subsection{Surface morphology:}

SEM images shows in figures which shows surface morphology of pure polyethylene glycol, crosslinked polyethylene glycol, Polyethylene glycol \& chitosan.

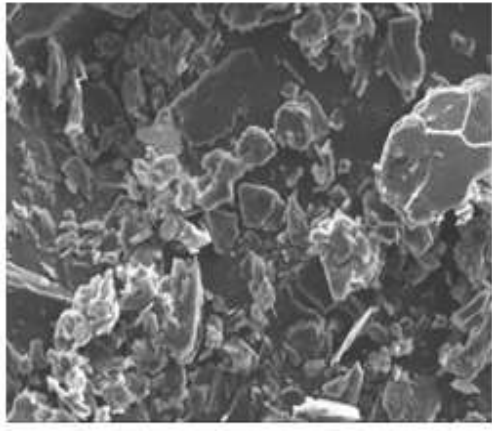

(a)

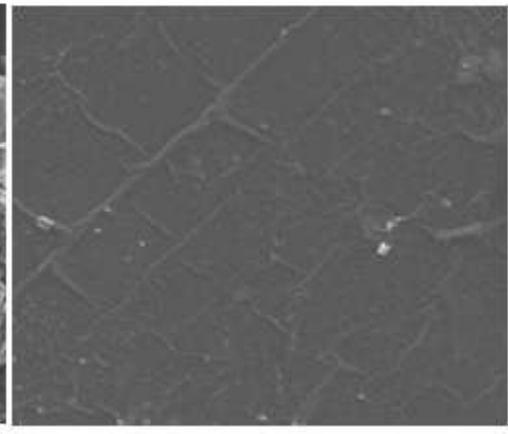

(b)

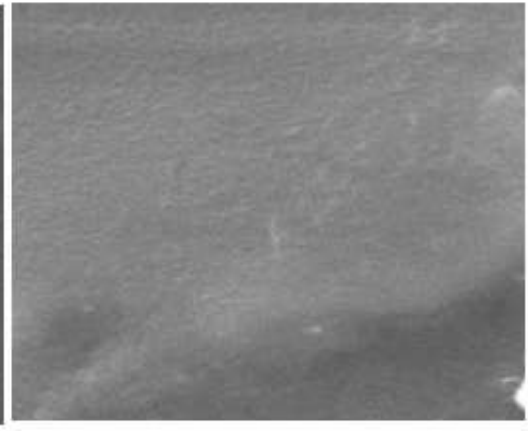

(c)

Fig.4. (a) SEM images of pure polyethylene glycol, (b) Crosslinked polyethylene glycol, (c) Polyethylene glycol \& chitosan conjugate

Polyethylene glycol \& chitosan have smoother surface than other two samples. It results strong antimicrobial properties.

\subsection{FTIR analysis:}

FTIR analysis represents adsorption peaks depend on the frequency of vibration of bonds of atom of that material. Size of peak denote amount of materials. Moon-Sung Kang et.al, 2006 explained that FTIR spectra of neat PEG and PEG crosslinked with GA [2]. With increasing the GA content, the $\mathrm{O}-\mathrm{H}$ stretching vibration band at near $3387 \mathrm{~cm}-1$ was decreased along with the band shift to a higher wave number at $3470-3432 \mathrm{~cm}-1$. This result suggests that the hydrogen bonding between - $\mathrm{OH}$ groups of PEG becomes weaker in crosslinked PEG than in neat PEG.

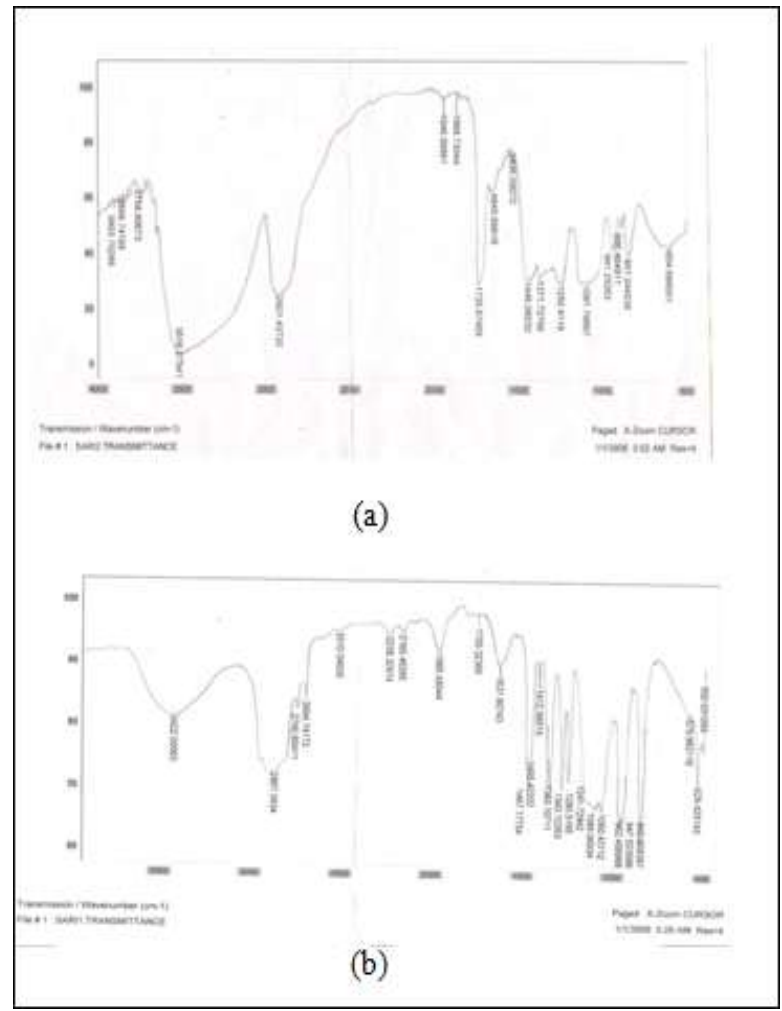

Fig. 5. (a) FTIR curve of pure polyethylene glycol, (b) FTIR curve of crosslinked polyethylene glycol
Table 1 wave number of FTIR analysis

\begin{tabular}{|l|l|l|}
\hline Bonding & $\begin{array}{l}\text { Reference wav } \\
\text { number }\end{array}$ & $\begin{array}{c}\text { Wave number } \\
\left(\mathrm{cm}^{-1}\right)\end{array}$ \\
\hline O-H bonding & 3387 & 3470 \\
\hline $\begin{array}{l}\text { C-O-C } \\
\text { bonding }\end{array}$ & 1298 & 1250.4119 \\
\hline C=O bonding & 1724 & 1735.67 \\
\hline
\end{tabular}

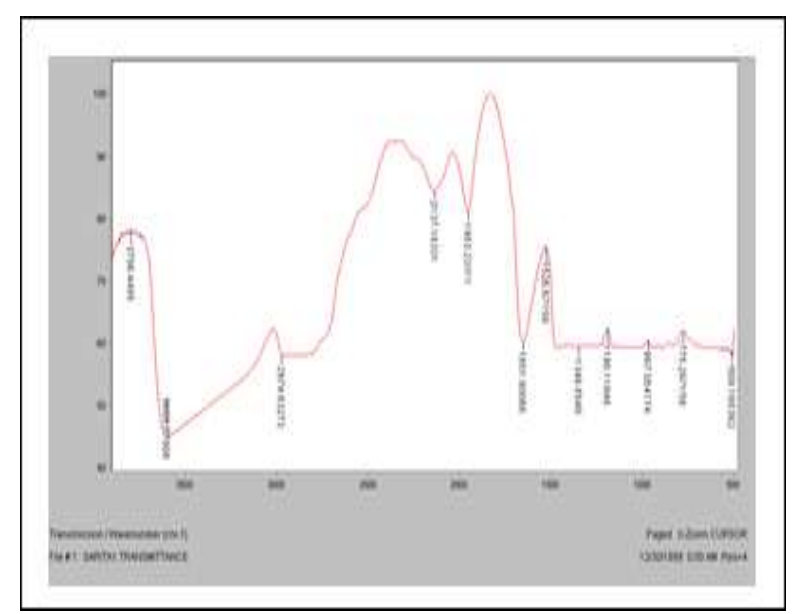

Fig. 6. FTIR curve of polyethylene glycol \& chitosan conjugate

The appearance of the aldehyde $\mathrm{C}=\mathrm{O}$ bond at 1735.67 $\mathrm{cm}-1$ with an addition of GA indicates that the aldehyde groups of GA do not completely react with $-\mathrm{OH}$ groups of PEG (i.e. non-functional reaction), and the unreacted aldehyde groups are also available for the coordination with other group [17].

In fig. 6 most of the characteristic peaks of PEG (1467, 1280, 1089, 962 and $842 \mathrm{~cm}^{-1}$ ) which is almost same to the polyethylene glycol \& chitosan. Since no appearance of new peaks or disappearance of peaks of individual components, it means that there was no chemical reaction between chitosan and PEG.

\section{Published By:}


Research on Polyethylene Glycol, Crosslinked Polyethylene Glycol \& Polyethylene Glycol Chitosan Conjugate Coating for Biomedical Application

\subsection{Mechanical testing:}

Crosslinked polyethylene glycol shows are good mechanical properties as compared to polyethylene glycol and chitosan conjugate film. When force is applied at stretched up breaks point after that film break this shows the breaks load of crosslinked polyethylene glycol. The mechanical properties of chitosan conjugate film are not good as compared to crosslinked polyethylene glycol film. When force is applied at stretched up breaks point after that film break this shows the breaks load.

The load bearing capacity depended on molecular weight of PEG [19]. The breaking point of PEG- PEG hydrogel was $10.5 \mathrm{~kg}$ and our result is $12.5 \mathrm{~kg}$. So by comparative study it concluded that crosslinked polyethylene glycol film have high weight bearing capacity. For crosslinked polyethylene glycol it is $12.5 \mathrm{~kg}$. Crosslinked polyethylene glycol has 4.4 $\%$ of elongation. Crosslinked polyethylene glycol has higher hardness as compared to polyethylene glycol \& chitosan. Polyethylene glycol \& chitosan conjugate sample shows not much good mechanical properties. Its mechanical strength is poor. The mechanical properties of chitosan and polyethylene glycol film can be improved by addition of crosslinker. Film of this conjugate is very soft that why it's not easy to done tensile testing on it.

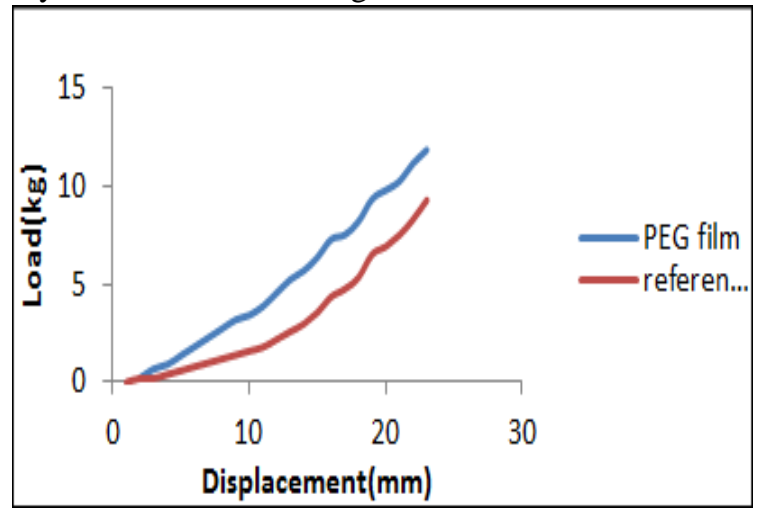

Fig. 7. Load V/S Displacement curve of Crosslinked PEG film

\subsubsection{Thickness:}

Thickness of film measured with Digital instrument. Film thickness is $0.217 \mathrm{~mm}, 0.302 \mathrm{~mm}, 0.373 \mathrm{~mm}$. Thickness value varied due to non uniformity.

Table 2 Thickness of coating films

\begin{tabular}{|c|c|}
\hline Sample & Thickness \\
\hline Crosslinked polyethylene glycol & $0.217 \mathrm{~mm}, 0.302 \mathrm{~mm}$, \\
\hline Polyethylene glycol \& chitosan & $0.058 \mathrm{~mm}, 0.060 \mathrm{~mm}$ \\
\hline
\end{tabular}

\subsubsection{Hardness}

Hardness of film is measured by Shore Hardness instrument. Hardness of crosslinked polyethylene glycol film is 75 - 82 shore.

\subsubsection{Breaking load}

Breaking load is the load at which film breaks. It's measured $12.5 \mathrm{~kg}$

\subsection{4. \% Elongation}

Value of \% elongation for polyethylene glycol film is 4.4

\section{CONCLUSION \& FUTURE WORK}

Chitosan and polyethylene glycol film is not mechanically strong. Mechanical properties of crosslinked polyethylene glycol are good as compared to other two samples. The mechanical properties of chitosan conjugate film are not good as compared to crosslinked polyethylene glycol film. For crosslinked polyethylene glycol it is $12.5 \mathrm{~kg}$. Crosslinked polyethylene glycol has $4.4 \%$ of elongation. Crosslinked PEG has higher hardness as compared to polyethylene glycol \& chitosan. Greater the amount of PEG more will be the strength of film. Surface morphology of all three samples it concluded that pure polyethylene is rough in surface as compared to crosslinked polyethylene glycol \& polyethylene glycol \& chitosan conjugate. SEM images showed that polyethylene glycol- chitosan surface was smoother as compared to pure PEG \& crosslinked PEG.

FTIR result showed that proper csosslinking of polyethylene glycol. FTIR results showed for polyethylene glycol $3422.00 \mathrm{~cm}^{-1}(\mathrm{O}-\mathrm{H}$ bond). DSC result of pure polyethylene glycol showed a high glass transition temperature than crosslinked polyethylene glycol \& polyethylene glycol \& chitosan conjugate. The different molecular weights of PEG had no significant influence on the mechanical properties and biocompatibility, perhaps because the molecular weight differences were not enough to significantly affect the material properties. DSC curve for pure polyethylene glycol showed sharp peak which show purity of sample but crosslinked polyethylene glycol and polyethylene glycol \& chitosan have broad peaks.

Comparative study of different concentration of polyethylene with conjugate to chitosan can be use for blending. Use lysozyme in polyethylene glycol for enhancing antimicrobial activity. Copolymers can be use as coating material. Use of Dextran in place of polyethylene glycol because it has high-density surface immobilization of biologically active molecules to low protein-binding surface coatings is desired. Mechanical properties of chitosan and polyethylene glycol can be increase by using crosslinker.

\section{REFERENCES}

1. M. Zhang. Properties and biocompatibility of chitosan films modified by blending with PEG. Biomaterials 23, 2002; 2641-2648.

2. Mohammad Reza Nejadnik. Polymer brush-coatings to prevent biomaterials associated infection Initial bacterial adhesion and biofilm formation May 2009.

3. Moon-Sung Kang. Dye-sensitized solar cells based on crosslinked poly (ethylene glycol) electrolytes. Journal of Photochemistry and Photobiology A: Chemistry 183, 2006; 15-21.

4. William D. Callister. Jr. Materials Science and Engineering an Introduction 8th edition.

5. Ling-hao He. Effects of blending chitosan with peg on surface morphology, Crystallization and thermal properties. Chinese Journal of Polymer Science Vol. 27 , No. 4, 2009; 501-510.

6. J.H. Kim, B.R. Min, K.B. Lee, J.Won, Y.S. Kang. Chem. Commun. 2002; 2732.

7. U. Seitz. Biliary stent clogging solved by 
nanotechnology? In vitro study of inorganic-organic sol-gel coatings for Teflon stents. Gastroenterology 133, 1,$2007 ; 65-71$.

8. Geetha Manivasagam et.al, Biomedical Implants: Corrosion and its Prevention - A Review, 2010

9. Todorka G. Vladkova, Surface Engineered Polymeric Biomaterials with Improved Biocontact Properties, 2010.

10. W. Senaratne, P. Sengupta, V. Jakubek, D. Holowka, C. K. Ober, and B. Baird. Functionalized surface arrays for spatial targeting of immune cell signalling. Journal of the American Chemical Society, vol. 128, no. 17, 2006; $5594-5595$.

11. J. Anderson. The future of biomedical materials, In Proceedings of a Forecast of the Future for Biomaterials, Imperial Colleage, September 2005.

12. Hidenon Otsuka. Functionalization of Polylactide (PLA) surface using Heterobifunctional PEGPLA Block Copolymers for the Control of Cell Behavior at Surfaces. 2000.

13. Baiyan Dong, Sorin Manolache , Amy C. L. Wong, Ferencz S. Denes. Antifouling ability of polyethylene glycol of different molecular weights grafted onto polyester surfaces by cold plasma 2010 .

14. Laura J. Suggs. Platelet adhesion on a bioresorbable poly (propylene Fumarate-co-ethylene glycol) copolymer. Biomaterials 20 (1999) 683-690.

15. L. Wolgemuth. The Performance and Suitability of Parylene Coating. Aug 2000, 42.

16. M. Reza Nejadnik. Adsorption of pluronic F-127 on surfaces with different hydrophobicities probed by quartz crystal microbalance with dissipation 2009.

17. Bhuvnesh Gupta \& Shalini Saxena. chitosan polyethylene glycol coated cotton membrane for wound dressing Indian journal of fibre and textile research. vol-36 September, 2011; 272-280.

18. Gui-Zhen Ke1, Ru-Ping Ruan1, Jin-Xiu Wang1, Wei-Dong Yu1. study on Hole Filling of Porous PU/PEG Membrane and Its Mechanical Properties. Journal of Fiber Bioengineering and Informatics Regular Article, Vol. 2 No. 4, 2010;

19. Liora almany. Biosynthetic hydrogel scaffolds made from fibrinogen and polyethylene glycol for $3 \mathrm{~d}$ cell cultures. biomaterials 26, 2005; 2467-2477. 